\title{
Association between matrix metalloproteinase-3 gene polymorphisms and tendon-ligament injuries: evidence from a meta-analysis
}

\author{
Rui Guo ${ }^{1}$, Aihaiti Aizezi ${ }^{1}$, Yong Fan ${ }^{1}$, Zhe $\mathrm{Ji}^{1}$, Wenzong $\mathrm{Li}^{2}$, Yongxian $\mathrm{Li}^{3}$, Zhigang Wang ${ }^{1 *}$ and Kai Ning ${ }^{{ }^{*}}$
}

\begin{abstract}
Background: Tendon-ligament injuries (TLIs), including Achilles tendinopathy, cruciate ligament injury, tennis elbow, rotator cuff injury, patellar tendinopathy, and tibial tendinopathy, are common musculoskeletal soft injuries during physical activity. Matrix metalloproteinase-3 (MMP-3) gene polymorphisms have been implicated in the etiology of TLIs in several genetic association studies with inconsistent results. The purpose of this study was to collect and synthesize the current evidences on the association of MMP-3 polymorphisms and TLIs.
\end{abstract}

Methods: The search was conducted using PubMed, Web of Science, EMBASE, Cochrane Library, CNKI and Wanfang databases, prior to July, 2021. Newcastle Ottawa Scale was used to appraise the study quality. Strengths of association were represented by odds ratios (ORs) and 95\% confidence intervals (95\% Cls).

Results: Thirteen studies with 2871 cases and 4497 controls met the eligibility criteria, and each study was in high quality. The overall analyzes suggested $r 33025058$ was associated with an increased TLIs risk (5A vs. 6A, OR=1.20,95\% Cl 1.03-1.40, $P=0.020$ ). However, the association was not found for $r 5679620$, rs591058, and rs650108 polymorphisms. Subgroup analysis by injury type suggested that $r 5679620$ polymorphism was associated with a reduced risk to Achilles tendon rupture ( $A A+A G$ vs. $G G, O R=0.46,95 \% \mathrm{Cl} 0.25-0.87, P=0.020$ ), and $r s 3025058$ was associated with an elevated risk to anterior cruciate ligament injury ( $5 \mathrm{~A} 5 \mathrm{~A}+5 \mathrm{~A} 6 \mathrm{~A}$ vs. $6 \mathrm{~A} 6 \mathrm{~A}, \mathrm{OR}=1.46,95 \% \mathrm{Cl} 1.03-2.06, P=0.030)$. When stratified by ethnicity, the findings indicated that $r 53025058$ polymorphism was associated with an increased TLIs risk among Caucasians (5A6A vs. 6A6A, $\mathrm{OR}=1.55,95 \% \mathrm{Cl} 1.09-2.42, P=0.020$ ) and Brazilians (5A5A vs. 5A6A +6A6A, $\mathrm{OR}=2.80,95 \% \mathrm{Cl} 1.44-5.45, P=0.002$ ).

Conclusion: Findings of this study suggest that rs679620 polymorphism is associated with a reduced Achilles tendon rupture risk, and rs3025058 polymorphism contributes to an increased TLIs risk in Caucasians and Brazilians. However, rs591058 and rs650108 polymorphisms do not show any association with TLIs.

Keywords: Matrix metalloproteinase-3, Polymorphism, Tendon-ligament injury, Meta-analysis

*Correspondence: rmyygr@126.com; 13579233099@163.com

1 Department of Orthopedic Center, People's Hospital of Xinjiang Uygur

Autonomous Region, No. 91 Tianchi Road, Urumqi 830001, Xinjiang, China

Full list of author information is available at the end of the article

\section{Background}

Tendons and ligaments within the upper and lower limbs, such as anterior cruciate ligament, rotator cuff tendon, patellar tendon, and Achilles tendon, are common sites of musculoskeletal soft tissue injuries during participating in physical activity [1]. It has been original author(s) and the source, provide a link to the Creative Commons licence, and indicate if changes were made. The images or other third party material in this article are included in the article's Creative Commons licence, unless indicated otherwise in a credit line to the material. If material is not included in the article's Creative Commons licence and your intended use is not permitted by statutory regulation or exceeds the permitted use, you will need to obtain permission directly from the copyright holder. To view a copy of this licence, visit http://creativecommons.org/licenses/by/4.0/. The Creative Commons Public Domain Dedication waiver (http://creativeco mmons.org/publicdomain/zero/1.0/) applies to the data made available in this article, unless otherwise stated in a credit line to the data. 
reported that the lifetime prevalence of tendon injuries was up to $23.9 \%$ among athletes and $5.9 \%$ in the general population [2]. Ligament injuries also occur in millions of individuals [3,4]. A considerable number of these affected individuals require surgery, imposing a heavy burden on society.

Both of tendons and ligaments are dense connective tissues which are composed of mesenchymal-derived cells [5]. Despite differing in anatomical locations and function, the two tissues are similar in basic components and molecular features [5]. Hence, it is conceivable that tendon-ligament injuries (TLIs) may share similar biological mechanisms.

Despite both extrinsic and intrinsic factors were identified predisposing to TLIs, the exact etiology of these injuries remains largely uncharacterized [6]. Genetic factors are suggested as intrinsic factors for TLIs. Familial studies have provided evidences that genetics may contribute to TLIs $[7,8]$. In recent years, high-throughput sequencing technique has resulted in substantial advance in the establishment of association between genetic markers and diseases. Genes like type I collagen alpha1 (COL1A1) [9, 10], type V collagen alpha1 (COL5A1) [11, 12], fibrillin-2 [13], matrix metalloproteinase-1 (MMP-1) [14, 15], and MMP-3 [16, 17] have been reported to be associated with TLIs. $M M P-3$ gene is one of the most extensively studied candidate genes.

MMP-3 protein, also known as transin/stromelysin, belongs to matrix metalloproteinases (MMPs) which are a cluster of zinc-dependent endopeptidases participating in the breakdown of extracellular matrix [18]. MMP-3 is encoded by $M M P-3$ gene, which is mapped to human chromosome 11q22.3. This endopeptidase possesses the function of degrading multiple substrates including fibronectin, laminin, collagens, and cartilage proteoglycans [19].

Raleigh et al. [20] initially reported that the GG genotype of rs679620 in MMP3 gene contributed to an increased risk to Achilles tendinopathy in Caucasians. Thus far, a number of studies have looked into $M M P-3$ polymorphisms and their impact on susceptibility to TLIs, with varying and inconclusive results. Some studies found that $M M P-3$ polymorphisms were significantly associated with TLIs $[16,17,21,22]$, while other studies obtained a null association [23, 24]. The inconformity may be caused by limited sample sizes, population stratification, clinical heterogeneity, and other factors. Therefore, the current evidence-based study was performed to gain a better understanding of the association of $M M P$ 3 polymorphisms and susceptibility to several common TLIs, including tendon injury, cruciate ligament injury, tennis elbow, rotator cuff injury, and so forth.

\section{Materials and methods}

\section{Literature search}

Literature search of this meta-analysis was from six databases including PubMed, Web of Science, EMBASE, Cochrane Library, China National Knowledge Infrastructure (CNKI), and Wanfang. All studies were published prior to July, 2021. No language restriction was set. The search strategy for PubMed database was: (Matrix metalloproteinase-3 OR MMP-3 OR MMP 3 OR Metalloproteinase 3, Matrix OR Transin OR MMP3 Metalloproteinase OR Stromelysin 1 OR Stromelysin) AND (SNP OR Mutation OR Variant OR Variation OR Polymorphism) and (Achilles tendon OR Tendon injury OR Achilles tendon pathology OR Achilles tendinopathy OR Achilles tendon rupture OR ACL injury OR Anterior cruciate ligament injury OR Ligament injury OR Anterior cruciate ligament tear OR ACL tear OR Tennis elbow OR Lateral epicondylitis OR Rotator cuff tear). This search strategy was transferred into corresponding search strategies in other databases. References in relevant reviews and full-text articles were screened to retrieve additional studies.

\section{Inclusion and exclusion criteria}

Studies were enrolled based upon the following criteria: (1) Comparative studies concerning the association of MMP-3 gene polymorphisms and TLIs; (2) TLIs were confirmed by clinical and/or imaging criteria; (3) Data on genotype frequency were reported to evaluate odds ratios (ORs) with 95\% confidence intervals (95\%CIs).

Correspondingly, the excluded criteria were: (1) The study did not satisfy the inclusion criteria; (2) Conference paper, review article, animal study, and case series; (3) Duplicate studies. If data were reported for more than once, the most comprehensive one was selected.

\section{Data extraction}

Two investigators (RG and AA), independently of each other, performed the data extraction according to a standardized form. The data extracted were: author's name, publication year, country or region, ethnicity, study design, detailed genotype frequency of cases and controls, and Hardy-Weinberg Equilibrium (HWE). A study with more than one independent cohort should be separated into several individual studies. In the event of any discrepancy, two investigators double-checked the articles together and resolved the discrepancy by discussion.

\section{Quality assessment}

Two investigators (RG and AA) respectively appraised the quality of eligible studies by using Newcastle Ottawa Scale (NOS) [25]. The NOS included three sections: 
selection, comparability, and exposure. For the "selection" and "exposure" categories, one point could be awarded for each item. For the "comparability" category, a maximum of two points could be awarded. A study was considered to be in high quality with $\geq 6$ scores. Disagreements between two investigators were resolved by discussion.

\section{Statistical analysis}

It was assumed that "V" was the variant allele, "W" was the wild allele, the genotypes of case and control subjects could be grouped into three types including VV, VW, and WW. In the current meta-analysis, five genetic models were investigated, including allele model (V vs. W), homozygous model (VV vs. WW), heterozygous model (VW vs. WW), dominant model (VV+VW vs. WW), and recessive model (VV vs. VW+WW). The strengths of the association were represented by using ORs and 95\% CIs. The effect sizes were described as significant if $P$ values $<0.05$. The heterogeneity between studies was determined using Q-statistical test and $I^{2}$ test. When considerable heterogeneity was observed $(P<0.10$ and $I^{2}>50 \%$ ), the effect size was combined with the randomeffects model; otherwise, the fixed-effects model was employed. Subgroup-analysis by injury type and ethnicity were performed.

\section{Sensitivity analysis and publication bias}

Sensitivity analysis was performed by sequentially excluding each individual study to appraise their influence on the ORs and 95\% CIs. Publication bias was examined by visual inspection of the symmetry of the funnel plots (the more symmetrical, the lower risk of publication bias). All statistical analyses were carried out using RevMan 5.3 software.

\section{Results}

\section{Literature search}

After the search of six databases, 86 potentially relevant items were identified, including 24 from PubMed, 25 from EMBASE, 29 from Web of Science, zero from Cochrane Library, one from Wanfang, six from CNKI, and one from other sources. Thirty-two duplicated records were removed after the initial screen. A further 36 records were excluded after screening the titles and abstracts. Of the remaining 18 items requiring full-text review, another five items were excluded with reasons (two records with insufficient data, one review, one record with irrelevant locus, and one conference abstract). Ultimately, thirteen original studies $[16,17$, 20-24, 26-31] fell within the scope of this meta-analysis. The flow diagram was presented in Fig. 1.

\section{Study characteristics and quality assessment}

Total participants, including 2871 cases and 4497 controls, could be grouped into Asians, Caucasians, and mixed population (Brazilians). All studies were published in English, and the publish year ranged from 2009 to 2020. Injury types in the original studies including rotator cuff tear (RCT) [17, 24, 26], Achilles tendinopathy (ATEN) [16, 20, 21, 27, 28], anterior cruciate ligament rupture (ACLI) [22, 23, 28, 31], Achilles tendon rupture (ARUP) [20, 27], patellar tendinopathy (PTEN) [30], and tibial tendinopathy (TTEN) [14]. All the included studies were case-control studies, with the exception of Haug et al.'s [30] cohort study. Except for the studies conducted by Assunção et al. [26] and Godoy-Santos et al. [14] on rs3025058 polymorphism, all the studies complied with HWE. Of note, the study by El Khoury et al. [27], Raleigh et al. [20], and Gibbon et al. [28] contained two, two, and three different case-control cohorts, respectively. The characteristics of each study were summarized in Table 1. According to NOS, all studies received $\geq 6$ scores, labeled as excellent quality (Table 2).

\section{Meta-analysis and subgroup analysis}

The strengths of the association of rs679620, rs591058, rs650108, and rs3025058 polymorphisms and TLIs risk were displayed in Table 3.

\section{Rs679620 polymorphism and TL/s}

Nine studies [16, 17, 20-23, 27, 28, 30] with 11 cohorts investigated the $r s 679620$ polymorphism and TLIs vulnerability, encompassing 2108 cases and 3896 controls. Significant between-study heterogeneity was examined in most contrasts. The combined data indicated that rs679620 polymorphism did not associate with TLIs under any genetic model (Fig. 2). Subgroup analysis by ethnicity suggested $r s 679620$ was not associated with TLIs in Caucasians. As only one study was conducted in Asians and Brazilians, subgroup analyses for these populations were not conducted. When stratified by injury type, it suggested that $r 5679620$ was associated with a reduced ARUP risk under heterozygous model (AG vs. GG, OR $=0.45,95 \%$ CI $0.23-0.87, P=0.020)$ and dominant model (AA+AG vs. GG, OR $=0.46,95 \%$ CI $0.25-$ $0.87, P=0.020$ ). However, no association was observed for ATEN and ACLI under any model.

\section{Rs591058 polymorphism and TL/s}

Five studies [16, 20,22, 28, 30] with seven cohorts examined the association of $r 5591058$ and TLIs, including 825 cases and 890 controls. All the participants were Caucasians. Because considerable heterogeneity was detected, the random-effects model was used. The 


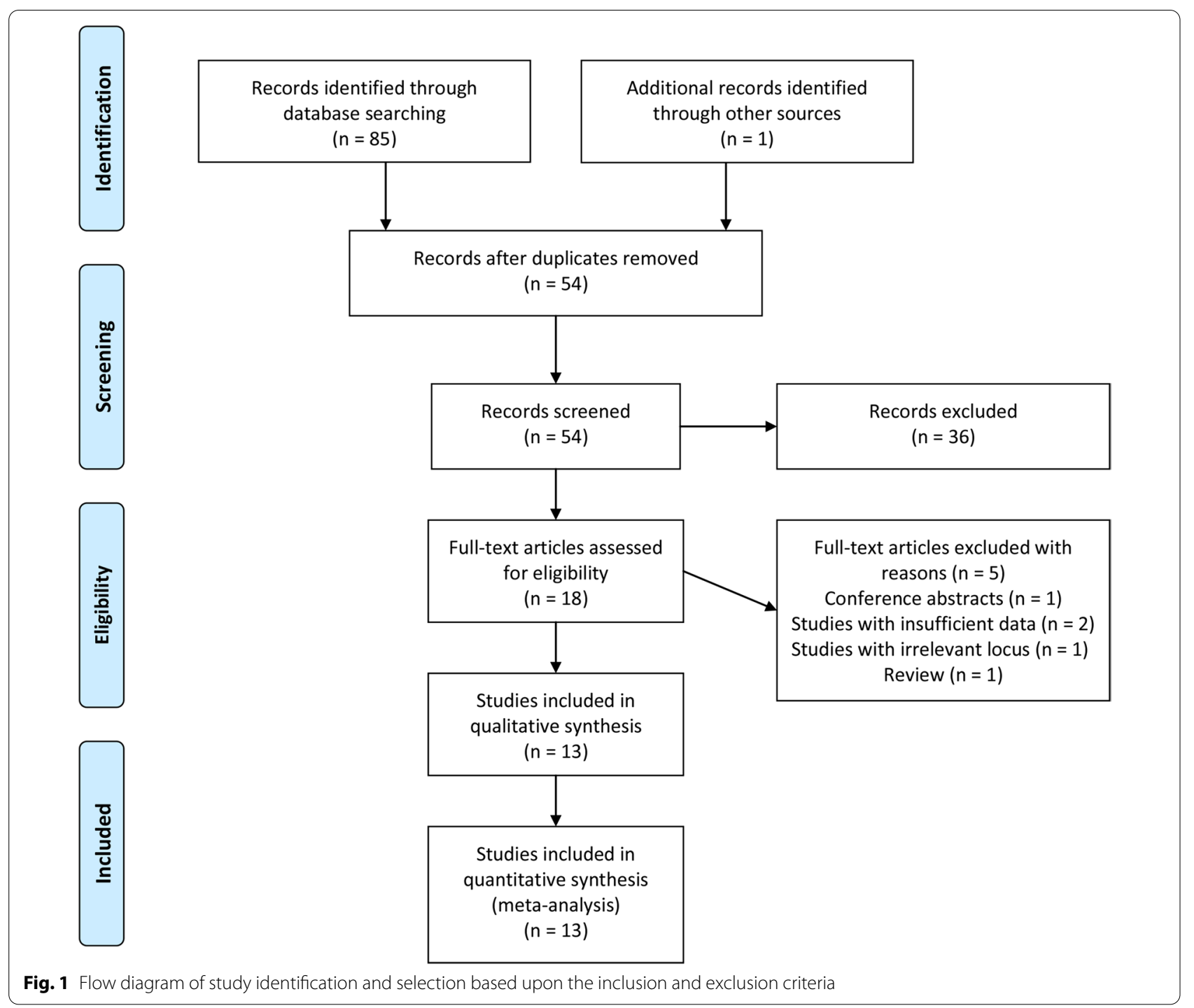

pooled data suggested that rs591058 was not associated with TLIs in the overall Caucasians. Subgroup analysis by injury type indicated that $r 5591058$ polymorphism was not associated with ATEN or ACLI.

\section{Rs650108 polymorphism and TLIs}

Four studies [16, 20, 28, 30] with six cohorts including 204 cases and 251 controls reported the association of rs650108 and TLIs. All the participants were Caucasians. For the overall Caucasians, the heterogeneity was not significant, and the fixed-effects model was used. The merged data suggested that $r s 650108$ was not associated with TLIs. Subgroup analysis by injury type also obtained a null association between $r s 650108$ polymorphism and ATEN.

\section{Rs3025058 polymorphism and TLIs}

Five studies [24, 26, 28, 29, 31] with seven cohorts reported the association of $r s 3025058$ and TLIs, including 412 cases and 325 controls. Significant heterogeneity was detected. Sensitivity analysis found the heterogeneity was mainly from the South African cohort of Gibbon et al.s study on ATEN [28], which could reverse the merged results. Therefore, Gibbon et al.s study was excluded. The combined data indicated rs3025058 was associated with an elevated TLIs risk under allele model (5A vs. 6A, OR $=1.20$, 95\% CI $1.03-$ $1.40, P=0.020$ ), homozygous model (5A5A vs. $6 \mathrm{~A} 6 \mathrm{~A}$, $\mathrm{OR}=1.48,95 \%$ CI $1.06-2.08, P=0.020$ ), heterozygous model $(5 \mathrm{~A} 6 \mathrm{~A}$ vs. $6 \mathrm{~A} 6 \mathrm{~A}, \mathrm{OR}=1.31,95 \%$ CI $1.03-1.67$, $P=0.030)$, and dominant model $(5 \mathrm{~A} 5 \mathrm{~A}+5 \mathrm{~A} 6 \mathrm{~A}$ vs. 6A6A, OR $=1.32,95 \%$ CI $1.05-1.67, P=0.020)$ in the overall population. 
Table 1 Main characteristics of included studies

\begin{tabular}{|c|c|c|c|c|c|c|c|c|c|c|c|c|}
\hline \multirow[t]{2}{*}{ Author } & \multirow[t]{2}{*}{ Year } & \multirow[t]{2}{*}{ Country/region } & \multirow[t]{2}{*}{ Ethnicity } & \multirow[t]{2}{*}{ Design } & \multirow[t]{2}{*}{ Diagnosis } & \multicolumn{3}{|l|}{ Case } & \multicolumn{3}{|c|}{ Control } & \multirow[t]{2}{*}{ HWE } \\
\hline & & & & & & VV & vw & WW & VV & vw & wW & \\
\hline Rs679620 & & & & & & AA & $A G$ & GG & $\mathrm{AA}$ & $A G$ & GG & \\
\hline Briški N & 2020 & Croatia & Caucasian & Case-control & ATEN & 16 & 30 & 17 & 9 & 51 & 32 & 0.08 \\
\hline El Khoury L (I) & 2016 & UK & Caucasian & Case-control & ATEN & 31 & 46 & 16 & 35 & 71 & 25 & 0.31 \\
\hline El Khoury L (II) & 2016 & UK & Caucasian & Case-control & ARUP & 6 & 8 & 11 & 35 & 71 & 25 & 0.31 \\
\hline Figueiredo EA & 2020 & Brazil & Mixed & Case-control & $\mathrm{RCT}$ & 41 & 111 & 58 & 90 & 275 & 200 & 0.78 \\
\hline Gibbon A (I) & 2017 & Australia & Caucasian & Case-control & ATEN & 20 & 40 & 17 & 39 & 94 & 58 & 0.94 \\
\hline Haug KBF & 2018 & Norway & Caucasian & Cohort & PTEN & 5 & 21 & 7 & 22 & 53 & 18 & 0.18 \\
\hline Lulinska-KuklikE & 2019 & Poland & Caucasian & Case-control & $A C L I$ & 54 & 107 & 68 & 59 & 93 & 40 & 0.77 \\
\hline Nie G & 2019 & China & Asian & Case-control & ATEN & 78 & 460 & 546 & 234 & 950 & 1004 & 0.68 \\
\hline Posthumus M & 2012 & South Africa & Caucasian & Case-control & $A C L I$ & 53 & 94 & 34 & 57 & 99 & 59 & 0.25 \\
\hline Raleigh SM (I) & 2009 & South Africa & Caucasian & Case-control & ATEN & 15 & 32 & 28 & 24 & 55 & 19 & 0.22 \\
\hline Raleigh SM (II) & 2009 & South Africa & Caucasian & Case-control & ARUP & 8 & 20 & 10 & 24 & 55 & 19 & 0.22 \\
\hline Rs591058 & & & & & & $\mathrm{TT}$ & TC & CC & $\mathrm{TT}$ & $\mathrm{TC}$ & $\mathrm{CC}$ & \\
\hline Briški N & 2020 & Croatia & Caucasian & Case-control & ATEN & 10 & 25 & 13 & 9 & 51 & 32 & 0.08 \\
\hline Gibbon A (I) & 2017 & Australia & Caucasian & Case-control & ATEN & 20 & 39 & 18 & 38 & 93 & 60 & 0.86 \\
\hline Gibbon A (III) & 2017 & South Africa & Caucasian & Case-control & $A C L I$ & 80 & 182 & 64 & 59 & 106 & 60 & 0.39 \\
\hline Haug KBF & 2018 & Norway & Caucasian & Cohort & PTEN & 5 & 21 & 7 & 22 & 51 & 20 & 0.35 \\
\hline Lulinska-KuklikE & 2019 & Poland & Caucasian & Case-control & $A C L I$ & 54 & 107 & 68 & 59 & 93 & 40 & 0.77 \\
\hline Raleigh SM (I) & 2009 & South Africa & Caucasian & Case-control & ATEN & 15 & 32 & 26 & 25 & 53 & 19 & 0.34 \\
\hline Raleigh SM (II) & 2009 & South Africa & Caucasian & Case-control & ARUP & 9 & 20 & 10 & 25 & 53 & 19 & 0.34 \\
\hline Rs650108 & & & & & & GG & GA & $\mathrm{AA}$ & GG & GA & $\mathrm{AA}$ & \\
\hline Briški N & 2020 & Croatia & Caucasian & Case-control & ATEN & 35 & 23 & 5 & 31 & 50 & 11 & 0.18 \\
\hline Gibbon A (I) & 2017 & Australia & Caucasian & Case-control & ATEN & 34 & 27 & 6 & 90 & 56 & 13 & 0.32 \\
\hline Gibbon A (III) & 2017 & South Africa & Caucasian & Case-control & ACLI & 205 & 99 & 15 & 122 & 90 & 12 & 0.38 \\
\hline Haug KBF & 2018 & Norway & Caucasian & Cohort & PTEN & 19 & 13 & 1 & 54 & 35 & 4 & 0.58 \\
\hline Raleigh SM (I) & 2009 & South Africa & Caucasian & Case-control & ATEN & 39 & 28 & 7 & 51 & 42 & 2 & 0.05 \\
\hline Raleigh SM (II) & 2009 & South Africa & Caucasian & Case-control & ARUP & 20 & 16 & 2 & 51 & 42 & 2 & 0.05 \\
\hline Rs3025058 & & & & & & $5 \mathrm{~A} 5 \mathrm{~A}$ & $5 A 6 \mathrm{~A}$ & $6 \mathrm{~A} 6 \mathrm{~A}$ & $5 \mathrm{~A} 5 \mathrm{~A}$ & $5 \mathrm{~A} 6 \mathrm{~A}$ & $6 \mathrm{~A} 6 \mathrm{~A}$ & \\
\hline Assunção JH & 2017 & Brazil & Mixed & Case-control & $\mathrm{RCT}$ & 15 & 38 & 11 & 4 & 44 & 16 & 0.01 \\
\hline Gibbon A (I) & 2017 & Australia & Caucasian & Case-control & ATEN & 20 & 40 & 17 & 40 & 93 & 57 & 0.86 \\
\hline Gibbon A (II) & 2017 & South Africa & Caucasian & Case-control & ATEN & 12 & 30 & 27 & 24 & 51 & 18 & 0.33 \\
\hline Gibbon A (III) & 2017 & South Africa & Caucasian & Case-control & ACLI & 77 & 185 & 64 & 57 & 108 & 60 & 0.55 \\
\hline Godoy-Santos AL & 2017 & Brazil & Mixed & Case-control & TTEN & 15 & 34 & 19 & 12 & 61 & 27 & 0.02 \\
\hline Malila S & 2011 & Thailand & Asian & Case-control & $\mathrm{ACLI}$ & 1 & 22 & 63 & 1 & 20 & 79 & 0.84 \\
\hline Miao K & 2019 & China & Asian & Case-control & $\mathrm{RCT}$ & 6 & 48 & 96 & 8 & 42 & 100 & 0.21 \\
\hline
\end{tabular}

$V$ variant allele, $W$ wild allele, HWE Hardy-Weinberg Equilibrium, ATEN achilles tendinopathy, ARUP achilles tendon rupture, ACLI anterior cruciate ligament injury, PTEN patellar tendinopathy, RCT rotator cuff tear, TTEN tibial tendinopathy

Subgroup analysis by ethnicity indicated $r s 3025058$ was associated with an increased TLIs risk in Caucasians and Brazilians. When stratified by injury type, it suggested that rs3025058 was associated with ACLI under heterozygous model and dominant model.

\section{Sensitivity analysis and publication bias}

The stableness and robustness of the results were estimated by sensitivity analysis. With sequential removal of an individual study from the analyses, no significant changes were observed in the re-calculated ORs and 95\%CIs, which confirmed the reliability of the results. Funnel plots did not exhibit obvious asymmetry, suggesting no significant existence of publication bias (Fig. 3).

\section{Discussion}

This meta-analysis suggested that rs679620 polymorphism was associated with a reduced ARUP risk, and rs3025058 polymorphism was associated with an increased TLIs risk in the Caucasians and Brazilians. 
Table 2 Quality assessment of included studies

\begin{tabular}{|c|c|c|c|c|c|c|c|c|c|}
\hline \multirow[t]{2}{*}{ Study ID } & \multicolumn{4}{|l|}{ Selection } & \multirow{2}{*}{$\begin{array}{l}\text { Control for } \\
\text { important } \\
\text { factor }\end{array}$} & \multicolumn{4}{|l|}{ Exposure } \\
\hline & $\begin{array}{l}\text { Adequate } \\
\text { definition of } \\
\text { cases }\end{array}$ & $\begin{array}{l}\text { Representativeness } \\
\text { of cases }\end{array}$ & $\begin{array}{l}\text { Selection } \\
\text { of control } \\
\text { subjects }\end{array}$ & $\begin{array}{l}\text { Definition } \\
\text { of control } \\
\text { subjects }\end{array}$ & & $\begin{array}{l}\text { Exposure } \\
\text { assessment }\end{array}$ & $\begin{array}{l}\text { Same } \\
\text { method of } \\
\text { ascertainment } \\
\text { for all subjects }\end{array}$ & $\begin{array}{l}\text { Non- } \\
\text { response } \\
\text { rate }\end{array}$ & Total \\
\hline $\begin{array}{l}\text { Assunção JH, } \\
2017\end{array}$ & $\star$ & 许 & $\star$ & $\star$ & $\star \star$ & $\star$ & $\star$ & $\star$ & 8 \\
\hline Briški N, 2020 & $\star$ & से & 糸 & $\star$ & $\star$ th & $\star$ & $\star$ & $\star$ & 7 \\
\hline $\begin{array}{l}\text { El Khoury L, } \\
2016\end{array}$ & $\star$ & 光 & $\star$ & $\star$ & $\star \star$ & $\star$ & $\star$ & $\star$ & 8 \\
\hline $\begin{array}{l}\text { Figueiredo } \\
\text { EA, } 2020\end{array}$ & $\star$ & it & 㑱 & $\star$ & $\star \star$ & $\star$ & $\star$ & $\star$ & 7 \\
\hline $\begin{array}{l}\text { Gibbon A, } \\
2017\end{array}$ & $\star$ & मे & $\star$ & $\star$ & $\star$ th & $\star$ & $\star$ & $\star$ & 7 \\
\hline $\begin{array}{l}\text { Haug KBF, } \\
2018\end{array}$ & $\star$ & $\star$ & $\star$ & $\star$ & $\star$ 类 & $\star$ & $\star$ & $\star$ & 8 \\
\hline $\begin{array}{l}\text { Lulinska-Kuk- } \\
\text { lik E, } 2019\end{array}$ & $\star$ & $\star$ & $\star$ & $\star$ & $\star$ 站 & $\star$ & $\star$ & $\star$ & 8 \\
\hline Malila S, 2011 & $\star$ & से & th & $\star$ & $\star \star$ & $\star$ & $\star$ & $\star$ & 7 \\
\hline Miao K, 2019 & $\star$ & it & $\star$ & $\star$ & $\star$ 放 & $\star$ & $\star$ & $\star$ & 7 \\
\hline Nie G, 2019 & $\star$ & it & $\star$ & $\star$ & $\star \star$ & $\star$ & $\star$ & $\star$ & 8 \\
\hline $\begin{array}{l}\text { Posthumus } \\
\text { M, } 2012\end{array}$ & $\star$ & से & $\star$ & $\star$ & $\star$ 放 & $\star$ & $\star$ & $\star$ & 7 \\
\hline $\begin{array}{l}\text { Raleigh SM, } \\
2009\end{array}$ & $\star$ & it & $\star$ & $\star$ & $\star$ 放 & $\star$ & $\star$ & $\star$ & 7 \\
\hline
\end{tabular}

However, rs591058 and rs650108 polymorphisms did not show any association with TLIs. TLIs are common musculoskeletal soft tissue injuries among athletes and physically active individuals. These injuries may prevent the affected individuals from achieving their full potential. Despite TLIs have been widely studied, the underlying mechanisms are still largely indeterminate. A better recognition of the mechanisms of TLIs may assist the prevention, treatment and rehabilitation of these injuries. Risk factors associated with TLIs are traditionally divided into intrinsic and extrinsic factors. Intrinsic risk factors like genetics may predispose individuals to a specific injury.

Polymorphisms within $M M P-3$ gene have previously been reported to be associated with several complex musculoskeletal disorders such as osteoarthritis [32], frozen shoulder [33], and disc degeneration [34]. As an intrinsic factor for TLIs, $M M P-3$ gene polymorphisms have received a lot of attention in the past years. Genetic association studies often statistically underpowered because of limited sample sizes. Therefore, the existing evidences were combined by a meta-analysis approach to increase the statistical power.

Tendons and ligaments are collagenous tissues with similar composition and structures. MMP-3, also known as stromelysin-1, are involved in the breakdown of collagenous matrix components [18]. It plays an essential role in the remodeling of connective tissues by mediating extracellular matrix homeostasis [35]. It is speculated that an increased expression of $M M P 3$ gene is of great importance to prevent pathological alterations in tendons [36]. Tendons with tendinopathy appear to have an elevated rate of matrix remodeling, hence making tendons more vulnerable to injury. Similarly, evidence has indicated that the biological activity of MMP-3 is reduced in ruptured tendons [37]. Clinical studies suggested that MMP-3 mRNA was down-regulated in Achilles tendinopathy tissues compared with control tissues $[36,38]$. All these evidences indicate that MMP-3 has an effect on TLIs risk.

Rs679620 variant is a non-synonymous polymorphism which leads to the substitution of a glutamate residue (GAA) by a lysine residue (AAA) of pro-MMP3. This alteration is speculated to influence the mature of MMP3 enzyme [20]. However, bioinformatics analyses by Nie et al. [21] suggested that this alteration could not significantly alter the biological function of the MMP3 protein. Therefore, the mechanisms underlying rs679620 polymorphism and TLIs still need to be investigated. In this study, significant association was observed between rs679620 and ARUP among Caucasians, but the included study number and sample size were quite limited. 
Table 3 Associations of matrix metalloproteinase 3 gene polymorphisms and tendon-ligament injuries

\begin{tabular}{|c|c|c|c|c|c|c|c|}
\hline \multirow[t]{2}{*}{ Genetic model } & \multicolumn{3}{|c|}{ Test of association } & \multirow[t]{2}{*}{ No. of cohorts } & \multicolumn{2}{|c|}{ Test of association } & \multirow{2}{*}{$\begin{array}{l}\text { Statistical } \\
\text { model }\end{array}$} \\
\hline & OR & $95 \% \mathrm{Cl}$ & $P$ & & $\mathrm{I}^{2}(\%)$ & $P$ & \\
\hline \multicolumn{8}{|l|}{ Rs679620 } \\
\hline \multicolumn{8}{|l|}{ A versus $G$} \\
\hline Overall & 0.97 & $0.81-1.17$ & 0.780 & 11 & 72 & $<0.001$ & $\mathrm{R}$ \\
\hline Caucasian & 0.96 & $0.76-1.21$ & 0.740 & 9 & 66 & 0.003 & $\mathrm{R}$ \\
\hline ATEN & 1.04 & $0.77-1.39$ & 0.810 & 5 & 76 & 0.002 & $\mathrm{R}$ \\
\hline ARUP & 0.70 & $0.47-1.04$ & 0.080 & 2 & 0 & 0.400 & $\mathrm{~F}$ \\
\hline $\mathrm{ACLI}$ & 0.95 & $0.56-1.64$ & 0.860 & 2 & 87 & 0.006 & $\mathrm{R}$ \\
\hline \multicolumn{8}{|l|}{ AA versus $G G$} \\
\hline Overall & 0.95 & $0.64-1.40$ & 0.790 & 11 & 73 & $<0.001$ & $\mathrm{R}$ \\
\hline Caucasian & 0.94 & $0.58-1.52$ & 0.810 & 9 & 66 & 0.002 & $\mathrm{R}$ \\
\hline ATEN & 1.10 & $0.56-2.14$ & 0.790 & 5 & 79 & $<0.001$ & $\mathrm{R}$ \\
\hline ARUP & 0.50 & $0.23-1.09$ & 0.080 & 2 & 0 & 0.550 & $\mathrm{~F}$ \\
\hline ACLI & 0.93 & $0.32-2.72$ & 0.890 & 2 & 87 & 0.006 & $\mathrm{R}$ \\
\hline \multicolumn{8}{|l|}{$A G$ versus $G G$} \\
\hline Overall & 0.92 & $0.71-1.20$ & 0.540 & 11 & 61 & 0.004 & $\mathrm{R}$ \\
\hline Caucasian & 0.84 & $0.57-1.23$ & 0.370 & 9 & 61 & 0.009 & $\mathrm{R}$ \\
\hline ATEN & 0.89 & $0.78-1.03$ & 0.120 & 5 & 46 & 0.120 & $\mathrm{~F}$ \\
\hline ARUP & 0.45 & $0.23-0.87$ & 0.020 & 2 & 50 & 0.160 & $\mathrm{~F}$ \\
\hline $\mathrm{ACLI}$ & 1.05 & $0.44-2.52$ & 0.910 & 2 & 84 & 0.010 & $\mathrm{R}$ \\
\hline \multicolumn{8}{|c|}{$A A+A G$ versus $G G$} \\
\hline Overall & 0.92 & $0.70-1.22$ & 0.580 & 11 & 70 & $<0.001$ & $\mathrm{R}$ \\
\hline Caucasian & 0.86 & $0.58-1.28$ & 0.460 & 9 & 68 & 0.002 & $\mathrm{R}$ \\
\hline ATEN & 0.95 & $0.65-1.40$ & 0.800 & 5 & 63 & 0.030 & $\mathrm{R}$ \\
\hline ARUP & 0.46 & $0.25-0.87$ & 0.020 & 2 & 37 & 0.210 & $\mathrm{~F}$ \\
\hline ACLI & 1.01 & $0.39-2.59$ & 0.990 & 2 & 88 & 0.004 & $\mathrm{R}$ \\
\hline \multicolumn{8}{|c|}{$A A$ versus $A G+G G$} \\
\hline Overall & 1.00 & $0.77-1.30$ & 1.000 & 11 & 57 & 0.009 & $\mathrm{R}$ \\
\hline Caucasian & 1.03 & $0.83-1.26$ & 0.810 & 9 & 40 & 0.100 & $\mathrm{~F}$ \\
\hline ATEN & 0.85 & $0.70-1.05$ & 0.130 & 5 & 77 & 0.002 & $\mathrm{R}$ \\
\hline ARUP & 0.84 & $0.43-1.65$ & 0.610 & 2 & 0 & 0.940 & $\mathrm{~F}$ \\
\hline $\mathrm{ACLI}$ & 0.89 & $0.55-1.46$ & 0.650 & 2 & 60 & 0.110 & $\mathrm{R}$ \\
\hline \multicolumn{8}{|l|}{ Rs591058 } \\
\hline \multicolumn{8}{|l|}{ T versus $C$} \\
\hline Overall & 0.96 & $0.76-1.20$ & 0.700 & 7 & 59 & 0.020 & $\mathrm{R}$ \\
\hline ATEN & 1.08 & $0.66-1.77$ & 0.760 & 3 & 75 & 0.020 & $\mathrm{R}$ \\
\hline $\mathrm{ACLI}$ & 0.90 & $0.59-1.37$ & 0.630 & 2 & 81 & 0.020 & $\mathrm{R}$ \\
\hline \multicolumn{8}{|l|}{ TT versus CC } \\
\hline Overall & 0.93 & $0.58-1.51$ & 0.780 & 7 & 61 & 0.020 & $\mathrm{R}$ \\
\hline ATEN & 1.25 & $0.43-3.59$ & 0.680 & 3 & 76 & 0.020 & $\mathrm{R}$ \\
\hline$A C L I$ & 0.83 & $0.36-1.93$ & 0.670 & 2 & 81 & 0.020 & $\mathrm{R}$ \\
\hline \multicolumn{8}{|l|}{ TC versus CC } \\
\hline Overall & 0.97 & $0.66-1.42$ & 0.860 & 7 & 57 & 0.030 & $\mathrm{R}$ \\
\hline ATEN & 0.91 & $0.44-1.8$ & 0.800 & 3 & 66 & 0.050 & $\mathrm{R}$ \\
\hline $\mathrm{ACLI}$ & 1.05 & $0.45-2.46$ & 0.910 & 2 & 86 & 0.008 & $\mathrm{R}$ \\
\hline \multicolumn{8}{|l|}{$\mathrm{TT}+\mathrm{TC}$ versus $\mathrm{CC}$} \\
\hline Overall & 0.95 & $0.66-1.41$ & 0.800 & 7 & 64 & 0.010 & $\mathrm{R}$ \\
\hline ATEN & 0.98 & $0.45-2.17$ & 0.970 & 3 & 75 & 0.020 & $\mathrm{R}$ \\
\hline ACLI & 0.97 & $0.41-2.27$ & 0.940 & 2 & 88 & 0.005 & $\mathrm{R}$ \\
\hline
\end{tabular}


Table 3 (continued)

\begin{tabular}{|c|c|c|c|c|c|c|c|}
\hline \multirow[t]{2}{*}{ Genetic model } & \multicolumn{3}{|c|}{ Test of association } & \multirow[t]{2}{*}{ No. of cohorts } & \multicolumn{2}{|c|}{ Test of association } & \multirow{2}{*}{$\begin{array}{l}\text { Statistical } \\
\text { model }\end{array}$} \\
\hline & OR & $95 \% \mathrm{Cl}$ & $P$ & & $I^{2}(\%)$ & $P$ & \\
\hline \multicolumn{8}{|l|}{$\mathrm{TT}$ versus $\mathrm{TC}+\mathrm{CC}$} \\
\hline Overall & 0.90 & $0.72-1.13$ & 0.370 & 7 & 28 & 0.220 & $\mathrm{~F}$ \\
\hline ATEN & 1.24 & $0.82-1.90$ & 0.310 & 3 & 48 & 0.150 & $\mathrm{~F}$ \\
\hline$A C L I$ & 0.81 & $0.61-1.08$ & 0.150 & 2 & 0 & 0.360 & $\mathrm{~F}$ \\
\hline \multicolumn{8}{|l|}{ Rs650108 } \\
\hline \multicolumn{8}{|l|}{ G versus A } \\
\hline Overall & 1.15 & $0.96-1.37$ & 0.140 & 6 & 44 & 0.110 & $\mathrm{~F}$ \\
\hline ATEN & 1.06 & $0.81-1.39$ & 0.670 & 3 & 70 & 0.040 & $\mathrm{R}$ \\
\hline \multicolumn{8}{|l|}{ GG versus $A A$} \\
\hline Overall & 1.03 & $0.64-1.67$ & 0.900 & 6 & 31 & 0.200 & $\mathrm{~F}$ \\
\hline ATEN & 0.85 & $0.24-2.92$ & 0.790 & 3 & 66 & 0.050 & $\mathrm{R}$ \\
\hline \multicolumn{8}{|l|}{ GA versus $A A$} \\
\hline Overall & 0.77 & $0.47-1.26$ & 0.300 & 6 & 0 & 0.520 & $\mathrm{~F}$ \\
\hline TEN & 0.71 & $0.36-1.39$ & 0.320 & 3 & 40 & 0.190 & $\mathrm{~F}$ \\
\hline \multicolumn{8}{|c|}{$\mathrm{GG}+\mathrm{GA}$ versus $\mathrm{AA}$} \\
\hline Overall & 0.91 & $0.57-1.46$ & 0.700 & 6 & 8 & 0.360 & $\mathrm{~F}$ \\
\hline ATEN & 0.77 & $0.28-2.13$ & 0.610 & 3 & 53 & 0.120 & $\mathrm{R}$ \\
\hline \multicolumn{8}{|c|}{ GG versus $G A+A A$} \\
\hline Overall & 1.26 & $1.00-1.57$ & 0.050 & 6 & 45 & 0.100 & $\mathrm{~F}$ \\
\hline ATEN & 1.21 & $0.62-2.36$ & 0.570 & 3 & 72 & 0.030 & $\mathrm{R}$ \\
\hline \multicolumn{8}{|l|}{ Rs3025058 } \\
\hline \multicolumn{8}{|l|}{$5 \mathrm{~A}$ versus $6 \mathrm{~A}$} \\
\hline Overall & 1.20 & $1.03-1.40$ & 0.020 & 6 & 0 & 0.740 & $\mathrm{~F}$ \\
\hline Caucasian & 1.16 & $0.95-1.42$ & 0.510 & 2 & 0 & 0.510 & $\mathrm{~F}$ \\
\hline Brazilians & 1.38 & $1.00-1.92$ & 0.050 & 2 & 0 & 0.340 & $\mathrm{~F}$ \\
\hline Asian & 1.12 & $0.80-1.56$ & 0.520 & 2 & 0 & 0.540 & $\mathrm{~F}$ \\
\hline $\mathrm{ACLI}$ & 1.14 & $0.91-1.42$ & 0.260 & 2 & 0 & 0.630 & $\mathrm{~F}$ \\
\hline $\mathrm{RCT}$ & 1.25 & $0.92-1.71$ & 0.150 & 2 & 50 & 0.160 & $\mathrm{~F}$ \\
\hline \multicolumn{8}{|l|}{$5 \mathrm{~A} 5 \mathrm{~A}$ versus $6 \mathrm{~A} 6 \mathrm{~A}$} \\
\hline Overall & 1.48 & $1.06-2.08$ & 0.020 & 6 & 0 & 0.350 & $\mathrm{~F}$ \\
\hline Caucasian & 1.37 & $0.91-2.08$ & 0.130 & 2 & 0 & 0.540 & $\mathrm{~F}$ \\
\hline Brazilians & 2.64 & $1.23-5.67$ & 0.010 & 2 & 0 & 0.180 & $\mathrm{~F}$ \\
\hline Asian & 0.83 & $0.30-2.30$ & 0.720 & 2 & 0 & 0.760 & $\mathrm{~F}$ \\
\hline $\mathrm{ACLI}$ & 1.27 & $0.78-2.05$ & 0.340 & 2 & 0 & 0.990 & $\mathrm{~F}$ \\
\hline $\mathrm{RCT}$ & 1.98 & $0.30-13.30$ & 0.480 & 2 & 79 & 0.030 & $\mathrm{R}$ \\
\hline \multicolumn{8}{|l|}{$5 \mathrm{~A} 6 \mathrm{~A}$ versus $6 \mathrm{~A} 6 \mathrm{~A}$} \\
\hline Overall & 1.31 & $1.03-1.67$ & 0.030 & 6 & 0 & 0.700 & $\mathrm{~F}$ \\
\hline Caucasian & 1.55 & $1.09-2.42$ & 0.020 & 2 & 0 & 0.790 & $\mathrm{~F}$ \\
\hline Brazilians & 1.95 & $0.55-1.67$ & 0.870 & 2 & 0 & 0.430 & $\mathrm{~F}$ \\
\hline Asian & 1.25 & $0.84-1.88$ & 0.280 & 2 & 0 & 0.730 & $\mathrm{~F}$ \\
\hline $\mathrm{ACLI}$ & 1.54 & $1.07-2.21$ & 0.020 & 2 & 0 & 0.710 & $\mathrm{~F}$ \\
\hline $\mathrm{RCT}$ & 1.21 & $0.78-1.86$ & 0.400 & 2 & 0 & 0.920 & $\mathrm{~F}$ \\
\hline \multicolumn{8}{|c|}{$5 \mathrm{~A} 5 \mathrm{~A}+5 \mathrm{~A} 6 \mathrm{~A}$ versus $6 \mathrm{~A} 6 \mathrm{~A}$} \\
\hline Overall & 1.32 & $1.05-1.67$ & 0.020 & 6 & 0 & 0.850 & $\mathrm{~F}$ \\
\hline Caucasian & 1.50 & $1.07-2.10$ & 0.020 & 2 & 0 & 0.970 & $\mathrm{~F}$ \\
\hline Brazilians & 1.17 & $0.68-2.00$ & 0.570 & 2 & 0 & 0.350 & $\mathrm{~F}$ \\
\hline Asian & 1.20 & $0.81-1.77$ & 0.360 & 2 & 0 & 0.640 & $\mathrm{~F}$ \\
\hline $\mathrm{ACLI}$ & 1.46 & $1.03-2.06$ & 0.030 & 2 & 0 & 0.840 & $\mathrm{~F}$ \\
\hline
\end{tabular}


Table 3 (continued)

\begin{tabular}{|c|c|c|c|c|c|c|c|}
\hline \multirow[t]{2}{*}{ Genetic model } & \multicolumn{3}{|c|}{ Test of association } & \multirow[t]{2}{*}{ No. of cohorts } & \multicolumn{2}{|c|}{ Test of association } & \multirow{2}{*}{$\begin{array}{l}\text { Statistical } \\
\text { model }\end{array}$} \\
\hline & OR & $95 \% \mathrm{Cl}$ & $P$ & & $I^{2}(\%)$ & $P$ & \\
\hline $\mathrm{RCT}$ & 1.22 & $0.81-1.85$ & 0.340 & 2 & 0 & 0.480 & $\mathrm{~F}$ \\
\hline \multicolumn{8}{|c|}{$5 \mathrm{~A} 5 \mathrm{~A}$ versus $5 \mathrm{~A} 6 \mathrm{~A}+6 \mathrm{~A} 6 \mathrm{~A}$} \\
\hline Overall & 1.36 & $0.85-2.19$ & 0.200 & 6 & 47 & 0.090 & $\mathrm{R}$ \\
\hline Caucasian & 1.01 & $0.72-1.41$ & 0.940 & 2 & 0 & 0.330 & $\mathrm{~F}$ \\
\hline Brazilians & 2.80 & $1.44-5.45$ & 0.002 & 2 & 16 & 0.280 & $\mathrm{~F}$ \\
\hline Asian & 0.78 & $0.29-2.15$ & 0.640 & 2 & 0 & 0.770 & $\mathrm{~F}$ \\
\hline ACLI & 0.92 & $0.62-1.35$ & 0.660 & 2 & 0 & 0.860 & $\mathrm{~F}$ \\
\hline $\mathrm{RCT}$ & 1.82 & $0.30-10.93$ & 0.510 & 2 & 80 & 0.020 & $\mathrm{R}$ \\
\hline
\end{tabular}

OR odds ratio, $C l$ confidence interval, $F$ fixed-effects model, $R$ random-effects model, ATEN Achilles tendinopathy, ARUP achilles tendon rupture, $A C L I$ anterior cruciate ligament injury, PTEN patellar tendinopathy, RCT rotator cuff tear, TTEN tibial tendinopathy

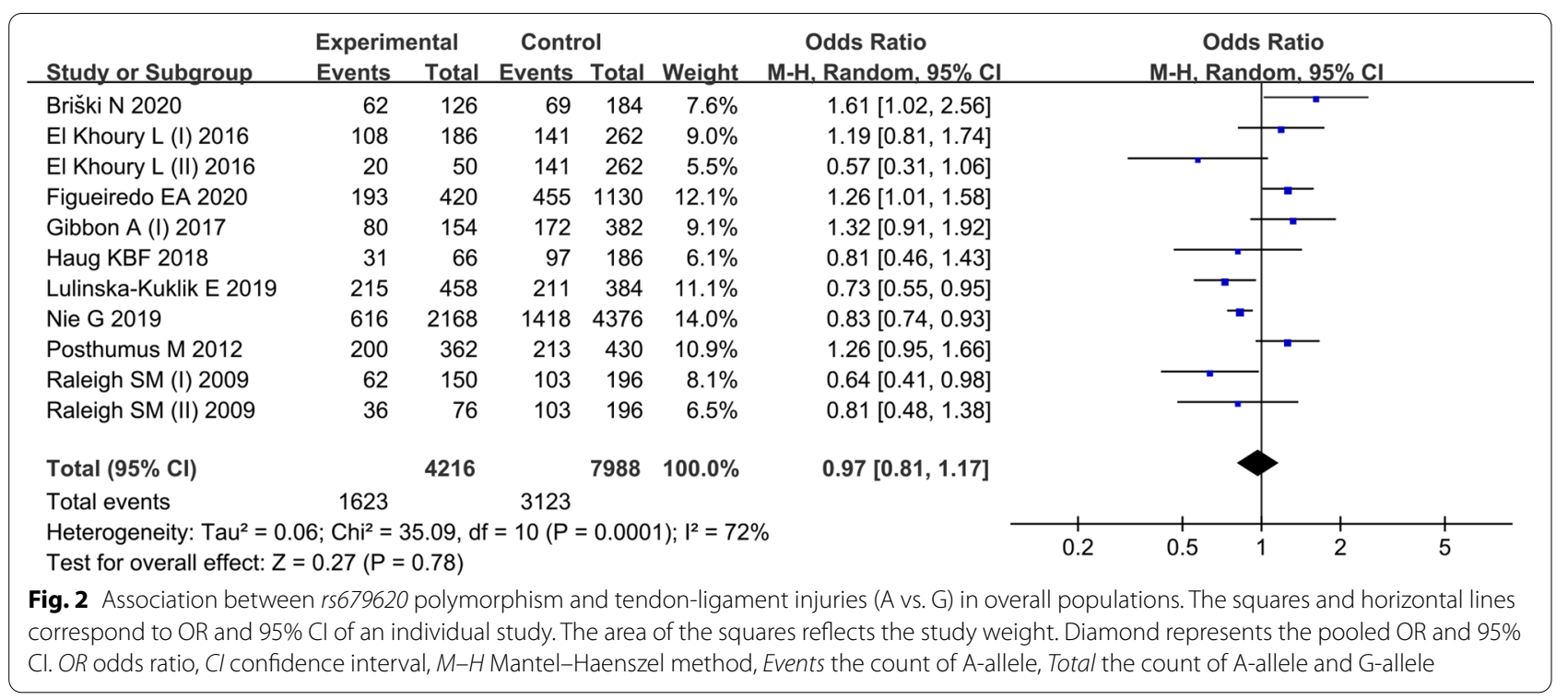

Further large sample studies are encouraged to verify this association.

Rs3025058 is featured by the presence of five or six adenines, leading to alleles $5 \mathrm{~A}$ and $6 \mathrm{~A}$. The $5 \mathrm{~A}$ allele was reported to have an elevated transcriptional activity compared with the $6 \mathrm{~A}$ allele of $M M P-3$ gene [39]. Literature has reported that higher production of MMP-3 in patients with RCT [40]. Rs591058 polymorphism is a $\mathrm{T} / \mathrm{C}$ transition at position 1547 situated in intron 4 , and rs650108 polymorphism is a G/A transition at position 495 within intron 8 . The functional of the two variants have not yet been established. It is reported that the two polymorphisms are in linkage disequilibrium with other polymorphisms within or beyond $M M P-3$ gene and form functional haplotypyes with influences on gene expressions and protein functions $[20,23]$.

This meta-analysis, despite being performed with a rigorous methodology, has limitations that should be addressed. Firstly, although subgroup analyses and sensitivity analyses were carried out, partial results should be interpreted with caution as a consequence of considerable heterogeneity. Secondly, the vast majority of included studies were retrospective case-control studies, future prospective cohort studies are encouraged to identify the causal association. Third, the cases enrolled in our present study were from different sport groups, which might result in an overestimation or underestimation of the conclusion. Fourth, the collective contribution of several associated polymorphisms and genes was not analyzed because of insufficient data. Last, for rs3025058 polymorphism, two studies [26, 29] were not in HWE, which might have some influence on the outcome. Therefore, more evidence should be considered from well-designed studies. 


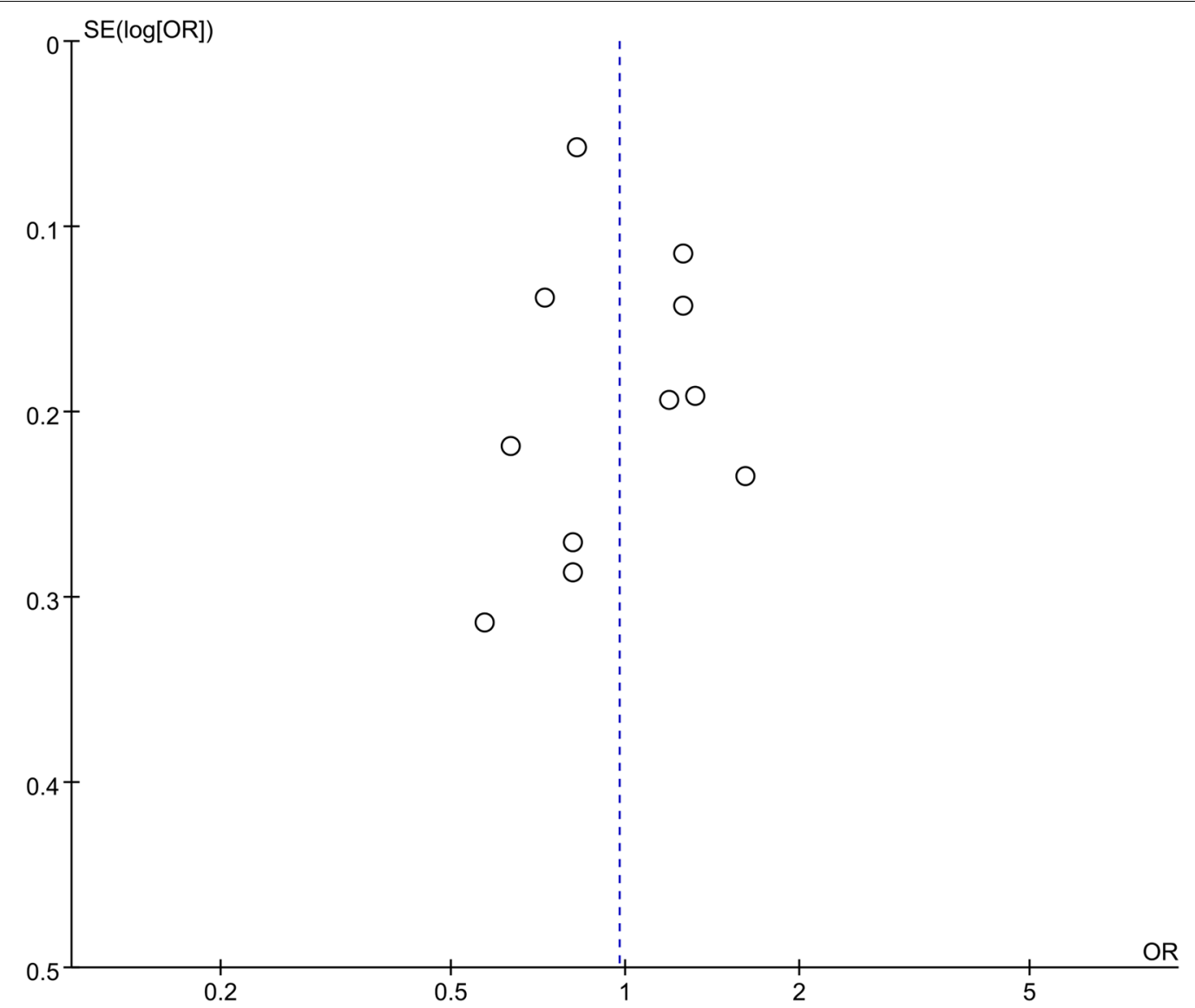

Fig. 3 Funnel plot analysis for publication bias of rs679620 polymorphism and tendon-ligament injuries (A vs. G) in overall populations. The funnel plot appears to be symmetrical, suggesting no significant publication bias

\section{Conclusion}

In summary, rs679620 polymorphism is associated with a reduced ARUP risk, and rs3025058 polymorphism contributes to an increased TLIs risk in Caucasians and Brazilians. However, rs591058 and rs650108 polymorphisms do not show any association with TLIs. Concerning limitations of this study, well-designed prospective cohort studies are encouraged to identify the association of $M M P-3$ polymorphisms and TLIs susceptibility.

\section{Acknowledgements}

Not applicable.

\section{Authors' contributions}

ZW and KN produced the idea of this study. RG and AA did the literature search, collected the data, analyzed the data, and drafted the manuscript. YF and $\mathrm{ZJ}$ interpreted the outcomes. WL and $\mathrm{YL}$ revised the manuscript. All authors read and approved the final version.

\section{Funding}

This work was supported by Natural Science Foundation of Xinjiang Uygur Autonomous Region (2018D01C120).

\section{Availability of data and materials}

The data used in this paper are available from the corresponding author upon reasonable request.

\section{Declarations}

Ethics approval and consent to participate

Not applicable.

\section{Consent for publication}

Not applicable.

\section{Competing interests}

The authors declare that they have no competing interests.

\section{Author details}

${ }^{1}$ Department of Orthopedic Center, People's Hospital of Xinjiang Uygur Autonomous Region, No. 91 Tianchi Road, Urumai 830001, Xinjiang, China.

${ }^{2}$ Department of Surgery, People's Hospital of Burgin County, Altay 836500, Xinjiang, China. ${ }^{3}$ Department of Surgery, People's Hospital of Tuoli County,

Tacheng 834300, Xinjiang, China.

Received: 13 November 2021 Accepted: 11 February 2022

Published online: 16 February 2022 


\section{References}

1. September AV, Schwellnus MP, Collins M. Tendon and ligament injuries: the genetic component. Br J Sports Med. 2007;41(4):241-6 (discussion 246)

2. Saini SS, Reb CW, Chapter M, Daniel JN. Achilles tendon disorders. J Am Osteopath Assoc. 2015;115(11):670-6.

3. Sanders TL, Maradit Kremers H, Bryan AJ, Larson DR, Dahm DL, Levy BA, Stuart MJ, Krych AJ. Incidence of anterior cruciate ligament tears and reconstruction: a 21-year population-based study. Am J Sports Med. 2016;44(6):1502-7.

4. Bram JT, Magee LC, Mehta NN, Patel NM, Ganley TJ. Anterior cruciate ligament injury incidence in adolescent athletes: a systematic review and meta-analysis. Am J Sports Med 2020:363546520959619.

5. Rumian AP, Wallace AL, Birch HL. Tendons and ligaments are anatomically distinct but overlap in molecular and morphological features-a comparative study in an ovine model. J Orthop Res. 2007;25(4):458-64.

6. Posthumus M, Collins M, September AV, Schwellnus MP. The intrinsic risk factors for ACL ruptures: an evidence-based review. Phys Sportsmed. 2011;39(1):62-73.

7. Caso E, Maestro A, Sabiers CC, Godino M, Caracuel Z, Pons J, Gonzalez FJ, Bautista R, Claros MG, Caso-Onzain J, et al. Whole-exome sequencing analysis in twin sibling males with an anterior cruciate ligament rupture. Injury. 2016;47(Suppl 3):S41-s50.

8. Flynn RK, Pedersen CL, Birmingham TB, Kirkley A, Jackowski D, Fowler PJ. The familial predisposition toward tearing the anterior cruciate ligament: a case control study. Am J Sports Med. 2005;33(1):23-8.

9. Khoschnau S, Melhus H, Jacobson A, Rahme H, Bengtsson H, Ribom E, Grundberg E, Mallmin H, Michaelsson K. Type I collagen alpha1 Sp1 polymorphism and the risk of cruciate ligament ruptures or shoulder dislocations. Am J Sports Med. 2008;36(12):2432-6.

10. Stepien-Slodkowska M, Ficek K, Zietek P, Kaczmarczyk M, Lubkowska W, Szark-Eckardt M, Cieszczyk P. Is the combination of COL1A1 gene polymorphisms a marker of injury risk? J Sport Rehabil. 2017;26(3):234-8.

11. Longo UG, Margiotti K, Petrillo S, Rizzello G, Fusilli C, Maffulli N, De Luca A, Denaro V. Genetics of rotator cuff tears: no association of col5a1 gene in a case-control study. BMC Med Genet. 2018;19(1):1-5.

12. Miyamoto-Mikami E, Miyamoto N, Kumagai H, Hirata K, Kikuchi N, Zempo H, Kimura N, Kamiya N, Kanehisa H, Naito H, et al. COL5A1 rs 12722 polymorphism is not associated with passive muscle stiffness and sports-related muscle injury in Japanese athletes. BMC Med Genet. 2019;20(1):192.

13. Khoury LE, Posthumus M, Collins M, van der Merwe W, Handley C, Cook J, Raleigh SM. ELN and FBN2 gene variants as risk factors for two sports-related musculoskeletal injuries. Int J Sports Med. 2015;36(4):333-7.

14. Godoy-Santos A, Cunha MV, Ortiz RT, Fernandes TD, Mattar R Jr, dos Santos MC. MMP-1 promoter polymorphism is associated with primary tendinopathy of the posterior tibial tendon. J Orthop Res. 2013;31(7):1103-7

15. Baroneza JE, Godoy-Santos A, Ferreira Massa B, Bocon de Araujo Munhoz F, Diniz Fernandes T, Leme Godoy dos Santos MC. MMP-1 promoter genotype and haplotype association with posterior tibial tendinopathy. Gene. 2014:547(2):334-7.

16. Briški N, Vrgoč G, Knjaz D, Janković S, Ivković A, Pećina M, Lauc G. Association of the matrix metalloproteinase 3 (MMP3) single nucleotide polymorphisms with tendinopathies: case-control study in high-level athletes. Int Orthop. 2021;45(5):1163-8.

17. Figueiredo EA, Loyola LC, Belangero PS, Campos Ribeiro-Dos-Santos AK, Emanuel Batista Santos S, Cohen C, Wajnsztejn A, Martins de Oliveira A, Smith MC, Pochini AC, et al. Rotator cuff tear susceptibility is associated with variants in genes involved in tendon extracellular matrix homeostasis. J Orthop Res. 2020;38(1):192-201.

18. Somerville RP, Oblander SA, Apte SS. Matrix metalloproteinases: old dogs with new tricks. Genome Biol. 2003;4(6):216.

19. Vaughn NH, Stepanyan H, Gallo RA, Dhawan A. Genetic factors in tendon injury: a systematic review of the literature. Orthop J Sports Med. 2017;5(8):2325967117724416.

20. Raleigh SM, van der Merwe L, Ribbans WJ, Smith RK, Schwellnus MP, Collins M. Variants within the MMP3 gene are associated with Achilles tendinopathy: possible interaction with the COL5A1 gene. Br J Sports Med. 2009;43(7):514-20.
21. Nie G, Wen X, Liang X, Zhao H, Li Y, Lu J. Additional evidence supports association of common genetic variants in MMP3 and TIMP2 with increased risk of chronic Achilles tendinopathy susceptibility. J Sci Med Sport. 2019;22(10):1074-8

22. Lulinska-Kuklik E, Rahim M, Moska W, Maculewicz E, Kaczmarczyk M, Maciejewska-Skrendo A, Ficek K, Cieszczyk P, September AV, Sawczuk M. Are MMP3, MMP8 and TIMP2 gene variants associated with anterior cruciate ligament rupture susceptibility? J Sci Med Sport. 2019;22(7):753-7.

23. Posthumus $M$, Collins $M$, van der Merwe $L, O$ 'Cuinneagain $D$, van der Merwe W, Ribbans WJ, Schwellnus MP, Raleigh SM. Matrix metalloproteinase genes on chromosome 11q22 and the risk of anterior cruciate ligament (ACL) rupture. Scand J Med Sci Sports. 2012;22(4):523-33.

24. Miao K, Jiang L, Zhou X, Wu L, Huang Y, Xu N, Zhang J, Li J. Role of matrix metalloproteases $1 / 3$ gene polymorphisms in patients with rotator cuff tear. Biosci Rep. 2019;39(10):BSR20191549.

25. Wells GA, Shea B, O'Connell D, Peterson J, Welch V, Losos M, Tugwell P. The Newcastle-Ottawa Scale (NOS) for assessing the quality of nonrandomised studies in meta-analyses. http://www.ohri.ca/programs/clini cal_epidemiology/oxford.asp.

26. Assunção JH, Godoy-Santos AL, Dos Santos M, Malavolta EA, Gracitelli MEC, Ferreira Neto AA. Matrix metalloproteases 1 and 3 promoter gene polymorphism is associated with rotator cuff tear. Clin Orthop Relat Res. 2017;475(7):1904-10.

27. El Khoury L, Ribbans WJ, Raleigh SM. MMP3 and TIMP2 gene variants as predisposing factors for Achilles tendon pathologies: attempted replication study in a British case-control cohort. Meta Gene. 2016;9:52-5.

28. Gibbon A, Hobbs H, van der Merwe W, Raleigh SM, Cook J, Handley CJ, Posthumus M, Collins M, September AV. The MMP3 gene in musculoskeletal soft tissue injury risk profiling: a study in two independent sample groups. J Sports Sci. 2017;35(7):655-62.

29. Godoy-Santos AL, Diniz Fernandes T, Massa BFS, de Cesar-Netto C, Santos MC, Rammelt S. Does MMP-3 polymorphism have a role in the etiology of the posterior tibial tendinopathy? Fuß \& Sprunggelenk. 2017:15(2):114-9.

30. Haug KBF, Visnes H, Sivertsen EA, Bahr R. Genetic variation in candidate genes and patellar tendinopathy: prospective cohort study of 126 elite volleyball players. Transl Sports Med. 2018;1(2):73-8.

31. Malila S, Yuktanandana P, Saowaprut S, Jiamjarasrangsi W, Honsawek S. Association between matrix metalloproteinase-3 polymorphism and anterior cruciate ligament ruptures. Genet Mol Res. 2011;10(4):4158-65.

32. Honsawek S, Malila S, Yuktanandana P, Tanavalee A, Deepaisarnsakul B, Parvizi J. Association of MMP-3 (-1612 5A/6A) polymorphism with knee osteoarthritis in Thai population. Rheumatol Int. 2013;33(2):435-9.

33. Chen W, Meng J, Qian H, Deng Z, Chen S, Xu H. A study of IL-1 beta, MMP-3, TGF-beta1, and GDF5 polymorphisms and their association with primary frozen shoulder in a Chinese Han population. Biomed Res Int. 2017;2017:3681645.

34. Yuan HY, Tang Y, Liang YX, Lei L, Xiao GB, Wang S, Xia ZL. Matrix metalloproteinase-3 and vitamin d receptor genetic polymorphisms, and their interactions with occupational exposure in lumbar disc degeneration. J Occup Health. 2010:52(1):23-30.

35. Foster BP, Morse Cl, Onambele GL, Ahmetov II, Williams AG. Genetic variation, protein composition and potential influences on tendon properties in humans. Open Sports Med J. 2012;6:8-21.

36. Ireland D, Harrall R, Curry V, Holloway G, Hackney R, Hazleman B, Riley G. Multiple changes in gene expression in chronic human Achilles tendinopathy. Matrix Biol. 2001;20(3):159-69.

37. Riley GP. Gene expression and matrix turnover in overused and damaged tendons. Scand J Med Sci Sports. 2005;15(4):241-51.

38. Alfredson H, Lorentzon M, Bäckman S, Bäckman A, Lerner UH. cDNAarrays and real-time quantitative PCR techniques in the investigation of chronic Achilles tendinosis. J Orthop Res. 2003;21(6):970-5.

39. Ye S, Eriksson P, Hamsten A, Kurkinen M, Humphries SE, Henney AM. Progression of coronary atherosclerosis is associated with a common genetic variant of the human stromelysin-1 promoter which results in reduced gene expression. J Biol Chem. 1996;271(22):13055-60.

40. Castagna A, Cesari E, Gigante A, Conti M, Garofalo R. Metalloproteases and their inhibitors are altered in both torn and intact rotator cuff tendons. Musculoskelet Surg. 2013;97(Suppl 1):39-47. 


\section{Publisher's Note}

Springer Nature remains neutral with regard to jurisdictional claims in published maps and institutional affiliations.

- fast, convenient online submission

- thorough peer review by experienced researchers in your field

- rapid publication on acceptance

- support for research data, including large and complex data types

- gold Open Access which fosters wider collaboration and increased citations

- maximum visibility for your research: over $100 \mathrm{M}$ website views per year

At BMC, research is always in progress.

Learn more biomedcentral.com/submissions 